\title{
Auf der Flucht vor den Nebenmenschen. Grillparzers \\ Reise nach Paris und London 1836
}

\author{
Gertrud Rösch
}

Als Grillparzer am 30. März 1836 in der Kutsche nach Paris fuhr, war er in halb erwünschter, hald unerwünschter Gesellschaft. Es begleiteten ihn zwei Kaufleute, in Linz stiegen zwei Gesellschaftsdamen ein, in München dann Hermine Elßler, die Cousine der in Paris lebenden Tänzerin Fanny Elßler, und ab Nancy saß noch ein französisches Ehepaar mit im Wagen.

Das Interesse an Berichten über diese und andere Reisen war bislang gering, seit August Sauer sie mit ausführlichen Anmerkungen in Abteilung II der historisch-kritischen Gesamtausgabe veröffentlichte ${ }^{1}$. Sie gehören zum biographischen und damit vernachlässigten Teil seines Oeuvre, weil es schwierig war, sie als ein Genre der Gebrauchsliteratur in die vorhandene Gattungstrias einzuordnen. Gerade die Gattungszuordnung war aber normativ für die Rezeption Grillparzers, dessen Dramen ungleich mehr Aufmerksamkeit fanden als seine Lyrik oder sein schmales erzählerisches Werk. Ebensosehr hängt diese Vernachlässigung innerhalb der Grillparzer-Forschung mit dem Unbehagen gegenüber dem Reisebericht zusammen, der erst in den sechziger und siebziger Jahren, nachdem Friedrich Sengle ihn als literarische Zweckform rehabilitiert hatte $^{2}$, in den Blick der Literaturwissenschaftler geriet. Aber selbst dann wurden vorzugsweise Berichte von Autoren wie Goethe, Heine oder Fontane untersucht, deren literarische Bedeutung sich auf andere Werke als die Reiseliteratur gründete. Seit den Arbeiten Friedrich Sengles ${ }^{3}$ haben die Untersuchungen zur Reiseliteratur vom Mittelalter bis zum zwanzigsten Jahrhundert, zur Kultur-, Ideen- und Gattungsgeschichte dieser vormals stiefmütterlich behandelten Textsorte stetig zugenommen. Der Forschungsüberblick Peter Brenners faßt die

1. Tagebuch auf der Reise nach Frankreich und England, in: Sämtliche Werke. Historisch-kritische Gesamtausgabe. Hrsg. von August Sauer. Abt. 2, Bd. 10: Tagebücher und literarische Skizzenhefte. Wien 1917, S. 3-135, Kommentar 346 403. Alle Zitate werden nach dieser Ausgabe mit der Abkürzung "Tgb." und der Nummer des Eintrags zitiert.

2. Friedrich Sengle, Die literarische Formenlehre. Vorschläge zu ihrer Reform. Stuttgart 1967, S. 10. (=Dichtung und Erkenntnis Bd.1).

3. Vgl. die Darstellung der Reisebeschreibung bei Friedrich Sengle, Biedermeierzeit. Deutsche Literatur im Spannungsfeld zwischen Restauration und Revolution 1815-1848. Bd. 2: Die Formenwelt. Stuttgart 1972, S. 238-278. 
Arbeiten zu dieser neu zu etablierenden Gattung zusammen und nennt auch den Gewinn, den die orthodoxe Germanistik aus der Analyse dieser Texte buchen könnte: "die Chance zur Erschließung vergangener oder fremder Kulturen und Mentalitäten, welche Form und Gehalt der Reiseberichte prägen". 4

Diese Erschließung von Mentalitäten und ihres Niederschlags im Text soll am Ende einer Untersuchung von Grillparzers Reisetagebüchern stehen. Zum Vergleich wird die Selbstbiographie herangezogen, um zu ermitteln, ob die Tendenzen der Tagebucheintragungen dort verstärkt oder zurückgedrängt werden; der zeitliche Abstand zwischen beiden Texten - Grillparzer verfaßte die Selbstbiographie eher zögernd und unwillig 1853 auf Wunsch der Österreichischen Akademie der Wissenschaften - legen hier Unterschiede nahe, deren Aussagekraft zu erfragen ist. ${ }^{5}$

Grillparzer unternahm vier größere Reise - 1819 nach Italien, 1826 nach Deutschland, 1836 nach Paris und London, 1847 nach Konstinopel und Griechenland. Die Tagebücher machen mehr als die Hälfte der autobiographischen Aufzeichnungen Grillparzers überhaupt aus.

Das Tagebuch ist einmal eine funktional bestimmte Gattung, eine Erinnerungshilfe. So verstand sie auch Grillparzer, der vier Tage nach seiner Ankunft in Paris notierte: "Gehe nach Hause und fange an diese ErinnerungsBehelfe niederzuschreiben" (Tgb. 2896). Darüber hinaus ist die Tagebuchform symptomatisch, denn darin spiegelt sich das Reisen, die Qualität des Vorgangs an sich. Reisen bedeutet die Begegnung des Ich mit der Welt, mit einer anderen, fremden, neuen Welt, die der Reisende mit einer bestimmten Absicht aufsucht; der Reisebericht hält diese Begegnung und ihre Nachwirkung fest: "Die 'erfahrene Welt' ist dem 'erinnerten Ich' auf eine selbstverständliche Weise zugeordnet". ${ }^{6}$

\section{II}

Die Fakten der Reise von 1836 lassen sich rasch ermitteln:

Am 30. März fuhr er von Wien ab, nach einem kurzen Aufenthalt in

4. Peter J. Brenner, Der Reisebericht in der deutschen Literatur. Ein Forschungsüberblick als Vorstudie zu einer Gattungsgeschichte. Tübingen 1990, S. $2(=2$. Sonderheft Internationales Archiv für Sozialgeschichte der deutschen Literatur).

5. Franz Grillparzer, Selbstbiographie, in: Sämtliche Werke. Ausgewählte Briefe, Gespräche, Berichte. Hrsg. von Peter Frank und Karl Pörnbacher. München 1965. Bd. 4, S. 20-178. Alle Zitate aus der Selbstbiographie sind aus dieser Ausgabe genommen.

6. Ralph-Rainer Wuthenow, Die erfahrene Welt. Europäische Reiseliteratur im Zeitalter der Aufklärung. Frankfurt 1980, S. 12. 
München kam er am 10. April in Paris an. Die Grenze hatte er bei Straßburg überschritten, wo ihm ein $\mathrm{PaB}$ ausgestellt wurde. Wiewohl er hinlänglich Französisch sprach, war es ein Ziel der Reise, seine Sprachkenntnisse noch zu vervollständigen. Auch das Führen eines Tagesbuchs gehörte zu den ausnahmsweise geübten Tätigkeiten, die aus der besonderen Situation resultierten. Im Brief vom 13. Mai an seinen Mitarbeiter Theodor Georg von Karajan - auf der ganzen Reise schrieb Grillparzer nur drei Briefe, zweimal an Katharina Fröhlich und diesen an Karajan - hob er ausdrücklich hervor, daß er mit dem Schreiben des Tagebuchs fast regelmäßig den Vormittag zubringe. ${ }^{7}$

Grillparzer wäre, wenn wir seiner Aussage gegenüber Karajan glauben können, als "premier poète de l'Allemagne" in vielen Häusern ein gem gesehener Gast gewesen, wenn man auch den Hinweis Karajans, Börne habe dafür gesorgt, daß Grillparzers Ankunft von drei Zeitungen berichtet worden sei, nicht verifizieren konnte ${ }^{8}$. Sein Verhalten beim Schließen von Bekanntschaften war zwiespältig. Einerseits mied er Begegnungen; unter den regelmäßigen Gesprächspartnern waren der schwedische Literaturhistoriker und Übersetzer Karl August Hagberg, den er aus Wien kannte, und die Familie Neuwall, deren Sohn Manuel Grillparzer mehrmals auf seinen Touren in der Stadt begleitete. Obwohl er ein Empfehlungsschreiben für den in Paris wohnenden James Maier Rothschild besaß, gab er es sehr spät ab, und dann nur, um die Adresse von Heinrich Heine zu erfahren und diesen zu besuchen. Er bedauerte immerhin, bei einem Frühstück mit dem deutschen Arzt Ferdinand Koreff, der seit.1822 in Paris lebte und mit Heine befreundet war, nicht Victor Hugo getroffen zu haben (Tgb. 3042). Dem jüngeren Dumas begegnete er mehrmals, ebenso den beiden Komponisten Rossini ( $T g b$. 3020) und Meyerbeer, deren Gesellschaft er sehr genoß; Die Hugenotten sah er nach eigener Aussage fünf- oder sechsmal (Bd. 4, S. 165). Die mehrfache Beteuerung im Tagebuch (Tgb. 3042), im Brief an Katharina Fröhlich vom 21. Mai $1836^{9}$ und in der Selbstbiographie, daß er literarische Bekanntschaften vermeiden wolle, ist somit leicht zu widerlegen.

In beiden Städten suchte er sich in der Politik zu orientieren; so erwähnte er den seit kurzem regierenden Louis Philippe mehrmals im Tagebuch lobend, wenn er ihn auch nie getroffen oder auf der Straße gesehen hatte: "Louis Philippe ist

7. An Karajan, in: Sämtliche Werke. Historisch-kritische Gesamtausgabe. Hrsg. von August Sauer. Abt. 3, Bd. 2: Briefe und Dokumente, 2. Teil. Wien 1924, S. 151-153.

8. Grillparzers Gespräche und Charakteristiken. Hrsg. von August Sauer. Bd. 3 (April 1831-März 1848), Wien 1906, S. 148, 481. (=Schriften des Literarischen Vereins in Wien Bd. 6).

9. An Katharina Fröhlich, in: Sämtliche Werke. Historisch-kritische Gesamtausgabe. Hrsg. von August Sauer. Abt. 3, Bd. 2: Briefe und Dokumente, 2. Teil. Wien 1924, S. 155-157. 
überhaupt ein Ehrenmann, und ein erzgescheiter Mann obendrein. Ich habe ihn vom ersten Augenblicke an dafür gehalten, und sehe hier nichts, was mich meine gute Meinung zurücknehmen ließe" ( $T g b$. 2906). Ähnlich heißt es am 5. Mai, nachdem der Namenstag des Königs mit Volksfest und Feuerwerk am 1. Mai gefeiert worden war:

Ich sehe immer mehr, der hiesige Zustand der Dinge ist befestigter als man bei uns glaubt. Nicht Louis Philipp und seine Dynastie. Man liebt ihn, oder vielmehr man ist der Uberzeugung, $\mathrm{da} B$ er für die Bedürfniße des Landes paßt. Er dürfte aber gewisse Gränzen überschreiten, an denen er schon hinstreift, und es wäre um ihn geschehen. (Tgb. 3009).

Grillparzer besuchte auch die Deputiertenkammer im Palais Bourbon, zu der er durch den österreichischen Gesandten Zutritt erhielt. Mit diesem Interesse an der politischen Lage stellte er sich in die Tradition der Reisenden zur Zeit der beiden Revolutionen, die sich durch einen Paris-Aufenthalt ein eigenes Bild von den vielbesprochenen Vorgängen in diesem Land machen wollten. Bei Grillparzer war dieses Bedürfnis aber schon zur Konvention erstarrt, wie sich am Eintrag vom 15. April ablesen läßt:

Wollte eine historische Tour, allein, machen. Den Platz der Bastille, den Temple sehen. Verfehlte die Direkzion und ging bis an die champs elysées ehe ich mein[en] Irrthum gewahr wurde. [...] Konnte den Platz der Bastille vor Müdigkeit nicht erreichen. (Tgb. 2898).

Diese Hinweise auf die Politik und sein eigenes Interesse daran unterschlägt Grillparzer in der Selbstbiographie. Dort spricht er nur von seinen Theaterbesuchen, die Ausgangspunkt zu abschätzigen Bemerkungen über die französischen Bühnen werden - im Tagebuch dagegen lobte er wiederholt die Aufführungen, die er sah und deren Theaterzettel er aufbewahrte. In der Selbstbiographie lautet der abschließende Kommentar: "Im übrigen habe ich in Paris gesehen, was jedermann sieht, es ist daher darüber nichts zu sagen.".

Dieser Selbsteinschätzung - "was jedermann sieht" - gegenüber ist Skepsis angebracht, denn sie ist Teil seiner Strategie, dieses Reiseerlebnis später zu verkleinern. Wichtig an diesem beiläufigen Satz ist jedoch die Berufung auf ein ihm und dem Leser gemeinsames Verständnis darüber, was jedermann in Paris sehen solle. Diese Konvention, was man gesehen haben muß, stellte der Reiseführer her, dessen Vorauswahl von berühmten Gegenständen Grillparzer vertraute. Gleich nach der Ankunft notierte er, daß er einen Plan der Stadt und einen Guide des Voyageurs kaufen wolle ( $T g b$. 2880), er tat es auch, nur um herauszufinden, daß ihm eine veraltete Ausgabe verkauft worden war (Tgb. 2881). 
Dieser Reiseführer - es war Le nouveau Conducteur de l'Étranger à Paris. Par F.M. Marchant. 18 e edition revue et augmentée par E. Hocquart. A Paris Jh. Moronval 1836, der in Grillparzers Bibliothek noch vorhanden ist - ging mit in die Notizen ein und war für deren Ausrichtung auf ausschließlich subjektive Eindrücke verantwortlich. Dies zeigen die Notate über seine Besichtigung am Mittwoch, den 13. April:

Hierauf über den pont neuf. Statue Heinrich IV. Wüßte nicht, daß sie mir sonderlich gefallen hätte. Der Kopf des Pferdes gut, das Übrige scheint mir nicht ausgezeichnet. Nôtre Dame etwas schwer, besonders mit dem Straßburger Münster verglichen. Im Innern, wie dieser, ausgeweißt und so verdorben. Die Priester sangen eben eine Art Vesper [...] Die Kirche muß man natürlich wiederholt besehen. Platz des zerstörten erzbischoflichen Pallastes. Aufs andere Ufer zurück. Hôtel de Ville. Kleiner als ich mirs vorgestellt hatte und es einer Stadt wie Paris ziemt. (Tgb. 2893).

Diese Monumente zu beschreiben oder zu erläutern, war nicht nötig, denn sie waren im Reiseführer abgebildet. ${ }^{10}$ Vielmehr bewertete der Tagebuchschreiber sie spontan nach persönlichen Vorlieben. Mehrmals mußte er zugeben, den Eindrücken, auf die ihn der Reiseführer lenkte, nicht gewachsen zu sein, so als er in die Nationalbibliothek ging. Dort wollte er zunächst nicht einmal deren deutschen Chefkonservator kennenlernen, sondern begnügte sich damit, einige leicht zugängliche Gegenstände zu besichtigen:

Ich benahm mich ganz wie der unwissende Reisende, den mein guide des voyageurs beschreibt und begaffte die Sachen, ohne mich um irgend etwas näher zu bekümmern. Theils sieht man derlei überall, theils verstehe ichs nicht, theils fehlt es mir an Zeit etwas zu approfondiren. ${ }^{11}$

Die gleichen Bedenken folgen, als er mit einem Deutschen über soziale Zustände spricht:

Details über den innern Verkehr, der durch nichts gehemmt wird. Fühle mich unglücklich, von solchen Dingen nichts zu verstehen, ja selbst die Erklärungen darüber nicht zu begreifen. ( $T g b .2954)$

Im Licht solcher Notate erscheint Grillparzers Aufenthalt zunehmend

10. Le nouveau Conducteur de l'Étranger à Paris. Par F.M. Marchant. 14e edition, Paris 1828. S. 67, 75, 181.

11. Tgb. 2982. Hagberg überredete ihn aber zu einem zweiten Besuch, bei dem sich Grillparzer dann das Ordnungssystem und einige Handschriften der Bibliothek zeigen ließ, vgl. $T g b .3017$. 
fragwürdig, denn zum einen scheint er vor Ort von der Erlebnissen schon überfordert, und zum anderen tilgt er später die herzlichen und erfrischenden Eindrücke aus dieser Stadt. Warum also reist er - die Antwort gibt er selbst:

Wäre froh, Paris wieder im Rücken zu haben. Was brauch' ich all das Zeug zu sehen und zu hören. Werde Wien wieder angenehm finden, wo ich wenigstens allein seyn kann. Wenn nur dort der schändliche Geistesdruck nicht wäre und die Erniedrigung der Nebenmenschen. ${ }^{12}$

\section{III}

In London verschärfte sich die in Paris beobachtete Fremdheit und Überforderung, denn Großbritannien war das Land,

dessen Sprache ich wohl im Lesen recht sehr gut verstehe, aus dem Munde eines Spechenden gehört aber nicht dem zehnten Wort nach verstehe, vielweniger daß ich sie irgend selbst spechen könnte ( $T g b$. 3042).

Am 17. Mai traf er dort ein, ausgestattet mit einem Empfehlungsschreiben, das ihn beim Londoner Zweig der Familie Rothschild einführen sollte, zudem eines an den Buchhändler John Murray, von dem er aber notierte: "Guter Gott! Mich in den Mittelpunkt der gelehrten Welt! Nahm übrigens den Brief, der wohl nie aus meinem Portefeuille herauskommen wird". ( $T g b$. 2957).

Er hatte also wie in Paris Empfehlungen und verschaffte sich auch Informationen auf die gleiche Weise: "Die Beamten sind höflich, betrügen mich aber, wie ich später sah, mit einem veralteten Plan von London und einem schlechten Guide de Voyageur". (Tgb. 3046).

Auch hier in London besuchte er vorwiegend die Theater, deren Programmzettel er wiederum aufbewahrte. Dieses Interesse an der Bühne wie die Ernüchterung über das ungebührliche Verhalten des Publikums, das Grillparzer in komischen Beispielen wiederholt im Tagebuch anprangerte, teilte er aber mit manchen zeitgenössischen Autoren, die ebenfalls die englische Metropole durch das Theater erlebten. ${ }^{13}$ Die Stadt selbst sah er, von Paris kommend, in steter

12. Tgb. 2953. Die gleiche Motivation trifft für andere Reisen zu, v.a. die nach Italien und Deutschland, deren Darstellung in der Selbstbiographie untersucht wurde von Arno Dusini, Die Ordnung des Lebens. Zu Franz Grillparzers "Selbstbiographie". Tübingen 1991, hier S. 99-102.

13. Roger Paulin, "Wilhelm von Burgsdorffs Reiseeindrücke von Paris und London 1798-1800 und 1817 und ihre Beziehungen zu Tiecks Englandbild", in: Rom Paris - London. Erfahrung und Selbsterfahrung deutscher Schriftsteller und Künstler in den fremden Metropolen. Ein Symposion. Hrsg. von Conrad 
Rückschau auf die französische Hauptstadt. Es überwogen die ungeheuren Dimensionen, die Grillparzer ständig betonte. ${ }^{14}$ Bei der Besichtigung einer Brauerei etwa berichtete er im Tagebuch:

Manche Einzelheiten kaum so groß als ich sie mir gedacht, das ganze Etablissement aber so riesenhaft, daß es einen schaudert. Beinahe alles durch eine Dampfmaschine verrichtet, die ziemlich unscheinbar, aber unermudlich ihren Weg geht und das verschiedenartigste durch denselben Mechanismus verrichtet. Gerstenvorräthe um eine belagerte Stadt zu proviantiren, Kühlapparate um darauf Schiffbruch leiden zu können [...]. (Tgb. 3088).

Vorher hatte er schon eine Dampfmaschinen-Druckerei besichtigt, über die er sich notierte: "Gefällig eingelassen, besehen wir das Ganze. Zauberartige Menschenthätigkeit der Maschine" (Tgb. 3077). Beide Male blieb Grillparzer gewissermaßen wortlos und hielt die Faszination der Maschine, nicht aber ihre revolutionäre Bedeutung für den menschlichen ArbeitsprozeB fest, die ihm wohl in diesem Augenblick kaum bewußt war. Dennoch bemerkte er die entschiedenen Eigenschaften, die nach Wolfgang Schivelbusch als die Grundelemente der Industrialisierung gelten können: "Gleichförmigkeit, Regelmäßigkeit, beliebige Dauer und Steigerung (Unermüdbarkeit)"15.

Die gleiche Feststellung über das Wesen der Maschine machte Grillparzer wieder am 31. Mai, als er das erste Mal mit der Eisenbahn fuhr:

Hierauf auf dem railway nach Deptford. Der ganze Weg in der Luft auf Bogenbrücken fortgeführt. 30 oder 40 Kutschen an einander gehängt erwarten den Dampfwagen, der sie in Bewegung setzen soll. Man steigt ein. Endlich verkündet ein Schnauben das rückkehrende Ungeheuer. Er wird vorgespannt. Nun stampft es und tobt es, die Bewegung erfolgt. Anfangs langsam dann rascher und rascher, bis das Ganze, ungefähr mit der Geschwindigkeit des Vogelfluges dahinstürmt. Die Schnelligkeit bemerkt man übrigens mehr an dem Vorüberfliegen der Gegenstände, als daß man im Wagen sitzend davon irgend affiziert würde. (Tgb. 3088)

In einer Nußschale faßte Grillparzer hier zusammen, was allen Zeitgenossen über die Eisenbahn, die zu diesem Zeitpunkt erst seit einem Jahr (die erste Bahn

Wiedemann. Stuttgart 1988, S. 585-594. Diskussionsbericht S. 683-685, hier S. 684. (=Germanistische Symposien-Berichtsbände Bd. 8)

14. Die Vergleiche, in denen er die Ausmaße der Stadt und ihrer Bauten zu fassen versuchte, sind gesammelt durch August Sauer, Vorbemerkung, in: Sämtliche Werke. Historisch-kritische Gesamtausgabe. Hrsg. von August Sauer. Abt. 2, Bd. 10: Tagebücher und literarische Skizzenhefte. Wien 1917, S. XII.

15. Wolfgang Schivelbusch, Geschichte der Eisenbahnreise. Zur Industrialisierung von Raum und Zeit im 19. Jahrhundert. München, Wien 1977, S. 15. 
zwischen Nürnberg und Fürth fuhr am 7. 12. 1835) in Deutschland existierte, bemerkenswert erschien: das veränderte Landschaftserlebnis, denn die Umgebung wird nur "vorüberfliegend", d.h. als Panorama wahrgenommen ${ }^{16}$.

Wo aber bleibt die Wertung dieser beiden Erlebnisse, in denen Grillparzer den Hauptakteur der Industrialisierung, die Dampfkraft, erlebte und, wenn auch unbewußt, deren entscheidende Eigenschaften erkannte - sie folgte, als er seinen Besuch im Britischen Museum aufschrieb. Über den Gang durch die Antikenabteilung meinte er:

O ewige Griechen! [...] London ist eine nicht üble Stadt, das Parthenon mag aber denn doch mehr werth gewesen seyn. Alles zerstört, aber uberall Spuren einer Schönheit, die man mit keinen Dampfapparaten herstellen und mit ihren höchsten Erzeugnissen nicht aufwiegen kann. ( $T g b$. 3090).

Wenige Tage später wiederholte er die gleiche Überlegung:

Durchlief den naturhistorischen Theil, der außer der Mineralogie, mit merkwürdigen Versteinerungen nichts Besonderes zu seyn scheint, und wendete mich wieder zu den Alterthümern, d.h. zu den Elgin Mamorn. Sog mich voll von den Eindrücken des Minerventempels. Diese Metopen, mehr als halb zerstört, und doch die Denkmäler der höchsten Schönheit. [...] O neue Pfeffer und Thee-Welt, wie kommst du da zur Vergleichung. ( $T g b .3095$ )

Wenig einträglich wäre es, Grillparzer sein Unverständnis und seine Skepsis im Hinblick auf die Technik als Mangel auszulegen. Viel gewinnbringender für eine mentalitätsgeschichtliche Einordnung solcher Äußerungen ist die Feststellung, daß ihm für die Technik geradezu die Worte fehlten: die Eisenbahn beschrieb er als Pferdekutsche, wie er sie kannte; ihre Funktionen wirkten auf ihn bedrohlich, wie die Worte aus dem Bereich des Dämonisch-Tierhaften es zeigen (Schnauben, Ungeheuer, stampft es, tobt es). Die Brauerei wiederum nahm eine Größe an, die ihm den Wortschatz der Katastrophe (Belagerung, Schiffbruch) abzwang. In der Bewunderung für die Antike schob er das Neue schließlich von sich.

Diese für seinen Bewußtseinsstand höchst aussagekräftigen Erlebnisse wurden in der Selbstbiographie von 1853 nicht erwähnt. Dort reihte er nur ärgerliche Episode in Anekdoten-Form aneinander, ohne den kulturgeschichtlichen Zusammenhang herzustellen, den er in der Rückschau durchaus gewinnen könnte, einen Zusammenhang, wie er ihn in der Autobiographie etwa für die politischen

16. Vgl. Peter J. Brenner, Der Reisebericht in der deutschen Literatur. Ein Forschungsüberblick als Vorstudie zu einer Gattungsgeschichte. Tübingen 1990, S. 492-497, hier S. $494 \quad(=2$. Sonderheft Internationales Archiv für Sozialgeschichte der deutschen Literatur). 
Folgen der jungdeutschen Literatur konstantiert. Aber gerade dank dieser Tatsache, $\mathrm{da}$ sich im Tagebuch offen ein staunendes Nichtbegreifen zeigt, darf man, zumindest was diese Reise von 1836 angeht, den Tagebüchern einen weitaus größeren Quellenwert zuschreiben als der Selbstbiographie.

Aber nicht nur im Hinblick auf Grillparzers Skepsis gegenüber der Technik sind die Tagebücher aussagekräftiger als der spätere Text, dies gilt auch für die literarischen Vorlieben, für deren Klärung das Tagebuch eine zweite verdeckte Aussage bereithält.

Über Heinrich Heine gibt er sich in den Tagebüchern wie der späteren Selbstbiographie eher lakonisch, und dies überrascht, da er doch so sehr die Begegnung wünschte:

Wir kamen gleich in die Literatur, fanden uns in unsem Neigungen und Abneigungen ziemlich auf demselben Wege und ich erfreute mich des seltenen Vergnügens bei einem deutschen Literator gesunden Menschenverstand zu finden. Er scheint durch die Bundestagsbeschlüße sehr alterirt und schrieb eben an einer Denkschrift an die abgeschmackte Versammlung. Vom Ultra-Liberalismus will er durchaus nichts wissen und spricht mit Verachtung von den deutschen Refugiés. Mit Börne steht er schlecht. Beklagt sich, daß dieser ihn für seinen Freund ausgegeben, was er nie gewesen. Gieng nach einer Stunde, herzlich entlassen. (Tgb. 2971).

In der Selbstbiographie wurde dieser positive Eindruck zurückgedrängt, ja Grillparzer zweifelte sogar Heines Urteil und persönliche Aufrichtigkeit an:

Bei Nennung meines Namens zeigte er große Freude und sagte mir viel Schmeichelhaftes, das er wahrscheinlich in der nächsten Stunde vergessen hat. [...] Er hatte aber mit Börne und überhaupt mit den selbst Verständigeren unter den Deutschen das gemein, daß er bei aller Mißbilligung des Einzelnen einen großen Respekt für das Ganze der deutschen Literatur hatte, ja sie allen andern voransetzte. Ich aber kenne kein Ganzes als welches aus Einzelnen besteht. Diesen aber fehlt der Nerv und der Charakter. (Bd. 4, S. 168).

Diese Verschiebung der Akzente vom Positiven des Tagebucheintrags zum Negativen der Selbstbiographie ist bei Ludwig Börne noch deutlicher. Am 14. April traf er ihn bereits bei Neuwalls und schrieb darüber: "Den Witz sieht man dem Manne wohl an, kaum aber die Gewalt, am wenigstens die Ausgelassenheit. Ich hatte mich herzlich auf ihn gefreut". (Tgb. 2897)

Er besuchte Börne zweimal in dessen Wohnung in Auteuil und bemerkte über 
das Zusammentreffen vom 20. April: "Das Gespräch wendete sich um staatsrechtliche Fragen, Politik, Literatur." (Tgb. 2924). Als dann zwei deutsche Verbannte eintraten und Börne Grillparzer und diese beiden sogar zum Mittagessen einladen wollte, lehnte der Besucher aus Wien ab und schrieb als Begründung:

theils weil ich heimische Klagen genug auf dem Herzen habe und keinen Beruf fühle, sie beim offenherzig machenden Glase Wein an Übertriebene [sic] gelangen zu lassen, theils weil ein Besuch bei Börne schon Stoff genug für einen Gesandtschaftsbericht ist, ein Mittagmahl aber gar, und noch dazu in solcher Gesellschaft, ohne Zweifel die Zahl der sieben Todsünden um eine achte vermehren würde. (Tgb. 2924).

In der Autobiographie wurden die Hinweise auf die sichtlich wohltuende Natur dieser Begegnung getilgt, vielmehr bewertete Grillparzer aus der Rückschau Börnes Person allein nach politischen Gesichtspunkten:

Börne war gewiß ein ehrlicher Mann und das politisch Aufreizende in seinen Schriften, . [...] kam wahrscheinlich nur daher, daß er die Deutschen für so dickhäutig hielt, daß man mit Prügeln dreinschlagen müsse, um nur die Spur eines geringen Eindruckes zurückzulassen. [...] Hätte er ein Jahr Achtundvierzig für möglich gehalten, er wäre etwa vorsichtiger gewesen. (Bd. 4, S. 167)

Zwei Schlußfolgerungen sind aus diesem Verhalten zu ziehen: Grillparzer folgte zum einen noch dem Paradigma "Dichterreise in die Nachbarschaft"17 und suchte Kontakt zu seinesgleichen auf die damals übliche Weise, durch Empfehlungen. Zum anderen geriet er in Paris mit Vertretern einer politischen Richtung in Berührung, die er ablehnte, sogar explizit in Bömes Gegenwart, dem er "gleich von Anfang an [s]eine gemäßigten Gesinnungen deutlich erklärte" ( $T g b$. 2924). In der Rückschau wurde die Ablehnung deutlicher gemacht, das Verhältnis kühler charakterisiert, als es nach Aussage des Tagebuchs war.

Das Unbehagen an den Autoren des "Jungen Deutschland" - der Bundestagsbeschluß, der diese griffige Formel prägte, lag kaum ein Jahr zurück ist in dem Reisetagebuch an einer anderen Stelle dokumentiert, wenn auch verdeckt. Die persönliche Zuneigung verhüllt nur scheinbar die literarische Gegensätzlichkeit. Schlüssel ist ein kurzer Text gleich zu Beginn der Aufzeichnungen, als Grillparzer den Zwischenaufenthalt in Linz beschreibt, wo zwei junge Frauen zusteigen.

17. Peter J. Brenner, Der Reisebericht in der deutschen Literatur. Ein Forschungsüberblick als Vorstudie zu einer Gattungsgeschichte. Tübingen 1990, S. 535-549. $(=2$. Sonderheft Internationales Archiv für Sozialgeschichte der deutschen Literatur). 
Da schlägt die Stunde und ich gehe querüber ins Posthaus, wo der Wagen schon bereit steht. Ich steige hinein womit folgende

Novelle

beginnt; blos Wahrheit, nicht Dichtung, weder der Form noch dem Inhalte nach.

Ich stieg in den Wagen, den der Kondukteur öffnete, und bleibe ein paar Sekunden im Tritte stehen. Nicht weil ich mit dem Mantel verwickelt, oder einen falschen Tritt gethan, oder daß mich meine Reise zu reuen angefangen hätte; vielmehr fieng sie mich erst jetzt an zu interessiren, denn mir gegenüber das Gesicht gegen die Pferde, saßen zwei der schönsten Frauenzimmer die. ich oder sonst Jemand irgend erblickt. [...] Das Gespräch war lebhaft, obgleich nicht interessant, über die Gegend an der Donau in der wir hinrollten, die doch alles übertreffe u.s.f. Es heißt der Linzer Weg, unterbrach die Blonde ihr Schweigen, und es gibt eine Menge Spitzbuben da, die die Leute umbringen oder sonst beschädigen. Wir zweifelten, sie beharrte aber auf ihren Spitzbuben, wobei ihr Organ und ihr Dialekt sich nicht auf die vortheilhafteste Art zeigten.

(Tgb. 2872-74).

Fortsetzung folgt.

Die Novelle, die keine ist, endet damit, daß die Blonde in München vier Stunden zu warten hätte, um die Kutsche nach Lindau zu erreichen. Auf diese Verlegenheit reagierten die Mitreisenden unterschiedlich:

Der Doktor empfahl sich, trotz des bedeutenden Blickes, den sie ihm zuwarf. Ich erboth mich ganz kalt, so lange bei ihr zu bleiben, bis ihre Sachen abgepackt seyn würden. Da trat der Schnurbart hervor und erklärte, diesen Dienst wolle er der Schönen leisten, eine Unterkunft im nächsten Gasthofe nehme er auf sich. Da empfahl ich mich und der Abgeschabte führte zum Lohn seines Ausharrens die Braut nach Hause. ( $T g b .2875)$.

Hält man nun gegen dieses Notat Grillparzers den folgenden Text, so fallen offensichtliche inhaltliche Parallelen ins Auge:

In Altenburg waren zwei Damen eingestiegen, und erst vor der Stadt hatten sie sich in die Gesichter gesehen und sich erkannt. Es schienen herzliche Freundinnen zu sein, die weit entfernt von einander gewesen waren. [...] Ich saß neben ihnen auf dem hintersten Sitz, und als es ganz finster war, und sie mich gewiß fest eingeschlafen glaubten, erzählte die eine mit leise flüsternder Stimme folgende Geschichte. Vorher hatte ich auf ihr Gespräch nicht Acht gehabt, der epische Ton aber, welcher plötzlich anhub, weckte mich alsbald. Die erzählende Dame hatte eine schöne Altstimme, welche zuweilen über das Flüstern heraustrat. Sonst sprach sie Alles ohne Modulation, und das erhöhte mir den Eindruck außerordentlich. [...]

Mein Weg führte über Schneeberg, der Postwagen ging aber gerade fort über Plauen nach Baiern hinein. Eh' ich meine verwirte Affekte geordnet hatte waren 
die Reiseeffekten geschieden, die Mädchen fuhren von dannen, ich hatte nicht den Mut gehabt, ein Wort an sie zu richten, hinaus in die Nacht fuhr das Mädchen mit dem süßen Gesicht meiner Jugendliebe. Ich stand schmerzlich bewegt; voll Trauer und Sehnsucht im Thorwege, und sah der Laterne des Wagens so lange nach, bis sie verschwand. ${ }^{18}$

Zwei Jahre vor Grillparzers Reisebericht hatte Heinrich Laube diese Erzählung einer Mitreisenden unter der Kapitelüberschrift "Die Novelle" im ersten Band seiner Reisenovellen erscheinen lassen. Dort erkennt der Ich-Erzähler in der reisenden Dame eine frühere Jugendliebe, gibt sich ihr aber nicht zu erkennen, sondern kostet die wehmütige Erinnerung an die frühere Beziehung aus. Einzelheiten aus Laubes Text kehren genau wieder, etwa die Angabe, auf welchen Platz in der Kutsche der Erzähler sitzt, um die beiden Frauen beobachten zu können, ferner der Hinweis auf deren attraktivens Aussehen. Andere Details werden ex negativo aufgenommen, so die angenehme Altstimme der Erzählerin bei Laube; Grillparzer bestätigt seiner Partnerin eine unangenehme Stimme. Vor allem aber arbeitet Grillparzer die frühere Liebesbeziehung in ihr Gegenteil um. Stehen bei Laube die aufgewühlten Gefühle im Vordergrund, bestätigt Grillparzer sich, ganz kalt geblieben zu sein; ebenso ablehnend betrug sich der zweite Mitreisende, der Doktor. Der schnurrbärtige Ungar hingegen bietet seine Hilfe an und darf auf Freundlichkeit und Dank der jungen Frau hoffen. Es kommt gerade keine Beziehung zustande, sondern ein Austausch von Gefälligkeiten, dessen Darstellung aber auf die Jugendliebe der Vorlage bezogen bleibt, denn die junge Frau wird "Braut" genannt. Der unordentlich gekleidete Fünfzigjährige ist weder attraktiv (vorher wird sein wenig ansprechendes Äußeres von Grillparzer ausführlich dargestellt) noch der gewünschte Partner (das wäre der sich empfehlende Doktor), sondern der Nutznießer einer Verlegenheit, und mit dieser Konstellation wird dem Motiv der Vorlage, der schwärmerischen Liebe, der entscheidende satirische Stoß versetzt. In diesem kurzen Text lieferte Grillparzer eine maliziöse und genaue Parodie auf Laubes Text, indem er einige Elemente aus der Vorlage übernimmt, damit diese Vorlage noch erkennbar bleibt, die zentrale Aussage aber in ihr Gegenteil verkehrt, mit der Absicht, das Modell lächerlich zu machen. Die Vorlage anzuerkennen und zugleich anzugreifen - das ist die Absicht der Parodie, die Grillparzer hier gelungen ist. ${ }^{19}$ Grillparzer kannte die Reisenovellen Laubes (im dritten, 1836 erschienenen Teilband widmete Laube dem Wiener Dramatiker ein ganzes Kapitel), denn er schrieb in den Erinnerungen an Beethoven (verfaßt

18. Heinrich Laube, Gesammelte Werke in 50 Bänden. Hrsg. von Heinrich Hubert Houben. Bd. 4: Reisenovellen I. Leipzig 1908, S. 68, 79f. (Zuerst 1834).

19. Andreas Höfele, "Parodie", in: Moderne Literatur in Grundbegriffen. Hrsg. von Dieter Borchmeyer und Viktor Žmegac. Frankfurt/M. 1987, S. 296-299. 
nach dem 14. 12. 1844) einige harte Worte über diese Gattung. Dort erwähnte er den geringen materiellen Gewinn aus seinen Werken und behauptete, sie seien zwar neben Uhland die besten Zeugnisse deutscher Dichtkunst, hätten ihm aber nicht soviel eingebracht "als einem Verstorbenen oder Lebendigen, oder HalbToten ein einziger Band ihrer Reisenovellen und Phantasiebilder". ${ }^{20}$ Dies ist ein leicht zu entschlüsselnder Hieb gegen drei Autoren: Hermann von PücklerMuskau, der nach England reiste, um eine Geldheirat in die Wege zu leiten und ab 1830 die auf diese Reise zurückgehenden "Briefe eines Verstorbenen" erscheinen ließ. Mit dem Lebendigen meinte Grillparzer Georg Herwegh, dessen "Gedichte eines Lebendigen" (erschienen 1841-43) die Briefe Pückler-Muskaus parodierten. Die Reisenovellen sind eben jene von Laube, die Grillparzer selbst parodiert.

Trotz der postulierten und durchaus glaubwürdigen Herzlichkeit gegenüber Heine wie Börne ist also die Gegnerschaft zu den jungdeutschen Autoren und deren bevorzugtem Genre Reisebeschreibung, dem Heine die entscheidenden Impulse gegeben hatte, im Tagebuch mehrmals präsent.

In Grillparzers Aufzeichnungen der Reise nach Paris und London berührten sich mehrere Traditionen des Erfahrens und Sehens. Einmal war der Wunsch nach Bildung noch präsent, denn er besuchte Museen und Galerien, ging ins Theater und wollte seine Sprachkenntnisse verbessern. Politische Kritik sowie Interesse an sozialen Verhältnissen scheinen durch, immer begleitet von Vergleichen mit den Zuständen in Wien und, in Paris, flankiert von der Furcht, durch Spitzel ausgehorcht und in Geheimberichten denunziert zu werden. Aber weder Schilderung von Politik und Gesellschaft noch die Darstellung des Literatur- und Theaterbetriebs sind Gegenstand des Tagebuchs. Vielmehr gerieten diese Gegenstände in lockerer Reihenfolge in den Blick eines Schreibers, der seine Notizen zu rein privaten Zwecken anlegte. Darin deutet sich die Zukunft der Gattung an, deren Informationswert auf eine andere Textsorte übergeht, den Reiseführer. Dessen Prototyp, Rheinreise von Straßburg bis Rotterdam von Karl Baedeker $^{21}$, war zum ersten Mal 1835 erschienen, ein Jahr vor Grillparzers Reise. Im Hinblick auf dessen Informationsfülle stellte sich die Frage, was die Reisenden selbst noch festhalten konnten, wenn die Details über Land und Leute schon

20. Erinnerungen an Beethoven, in: Sämtliche Werke. Ausgewählte Briefe, Gespräche, Berichte. Hrsg. von Peter Frank und Karl Pömbacher. Bd. 4. München 1965, S. 200.

21 Peter J. Brenner, Der Reisebericht in der deutschen Literatur. Ein Forschungsüberblick als Vorstudie zu einer Gattungsgeschichte. Tübingen 1990, S. 341. (=2. Sonderheft Interantionales Archiv für Sozielgeschichte der deutschen Literatur). 
gedruckt vorlagen. Buchenswert blieben dann nur noch die privaten Reaktionen und Erlebnisse. Letztendlich führt eine derartige Entwicklung zur "Schematisierung des Reisens" und "Ent-Individualisierung des Erlebens". Ob man deswegen schon von einem "Niedergang der Reisetagebücher" 22 sprechen sollte, bleibt zu bezweifeln. Eher scheint es, als würde das Tagebuch auf seinen privaten Ursprung zurückgeführt, an dem es die Aufgabe hat, die Reaktionen des Reisenden auf die neue Umgebung festzuhalten. Gerade dank seiner Form, der rein persönlichen Aufzeichnung, ist Grillparzers Reisetagebuch, ungeachtet der darin spürbaren konservativen Animosität gegen die zeitgenössische Literatur und die Technik, also höchst charakteristisch für seine Zeit.

22. Herbert Jost, Selbstverwirklichung und Seelensuche. Zur Bedeutung des Reiseberichts im Zeitalter des Massentourismus, in: Der Reisebericht. Die Entwicklung einer Gattung in der deutschen Literatur. Hrsg. von Peter J. Brenner. Frankfurt/M. 1989, S. 490-507, hier S. 492. (stm 2097). 